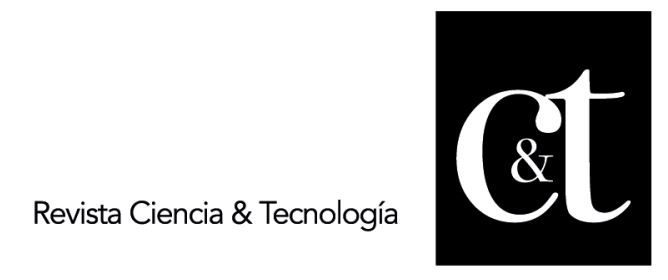

No. 33, 31 de enero de 2022

ISSN impreso: 1390 - 6321

ISSN online: 2661 - 6734

\title{
Potencialidad de la agricultura traspatio de precisión como estrategia táctica en la reactivación: mujeres mexicanas
}

\section{Potential of precision backyard agriculture as a tactical strategy in reactivation: Mexican women}

\author{
Nayeli Montalvo-Romero ${ }^{1}$ \\ naiemontalvor@gmail.com \\ https://orcid.org/0000-0002-3706-6244 \\ Aarón Montiel-Rosales ${ }^{2}$ \\ ar.chimedes@hotmail.com \\ https://orcid.org/0000-0002-3064-0274 \\ Ana Karen Merino-Torres ${ }^{3}$ \\ ana_karen_mt@hotmail.com \\ José Luis Tavares-López ${ }^{4}$ \\ jose.luis.tavares.lopez@outlook.com
}

Recibido: 28/9/2021, Aceptado: 28/12/2021

\begin{abstract}
RESUMEN
Las Naciones Unidas han proyectado que el impacto devastador sobre el desarrollo socioeconómico derivado de la pandemia por COVID-19 tendrá un efecto a largo plazo. En América Latina y el Caribe (ALyC), según la Comisión Económica para América Latina y el Caribe (CEPAL) la economía de la región crecerá un $5.2 \%$ en 2021. La CEPAL, menciona que para el 2022 solo se llegará al $2.9 \%$, incrementando con ello la pobreza extrema -factor determinante en el incremento del hambre-. En el presente artículo, se presenta como la agricultura traspatio permite abastecer de alimento para autoconsumo en las familias mexicanas, permitiendo que el excedente - sí, es que existe-, sea comercializado; permitiendo generar un ingreso económico a la familia. El alcance del estudio es de tipo exploratorio sobre los hábitos de las familias del Bajío; del estudio realizado se desprende que son las mujeres, quienes impulsan el cultivo de hortalizas y frutales, para obtener alimentos sanos y frescos; y en tal sentido, abonar a garantizar la seguridad alimentaria. A partir de lo anterior, se desprende que la agricultura traspatio de precisión impulsado por las mujeres mexicanas es una estrategia táctica viable, que abona a la reactivación socioeconómica en tiempos post COVID-19.
\end{abstract}

\footnotetext{
${ }^{1}$ División de Ingeniería Industrial, Tecnológico Nacional de México / ITS de Purísima del Rincón, México.

2 División de Ingeniería Industrial, Tecnológico Nacional de México / ITS de Purísima del Rincón, México.

${ }^{3}$ División de Ingeniería Industrial, Tecnológico Nacional de México / ITS de Purísima del Rincón, México.

${ }^{4}$ División de Ingeniería en Sistemas Automotrices, Tecnológico Nacional de México / ITS de Purísima del Rincón, México.
} 


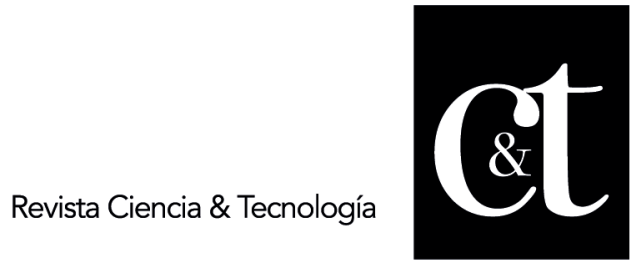

No. 33, 31 de enero de 2022

ISSN impreso: 1390 - 6321

ISSN online: 2661 - 6734

Palabras clave: Agricultura traspatio, mujeres campesinas, reactivación socioeconómica, post COVID-19, seguridad alimentaria.

\begin{abstract}
The United Nations has projected that the devastating impact on socioeconomic development resulting from the COVID-19 pandemic will have a longterm effect. In Latin America and the Caribbean (ALyC), according to the Economic Commission for Latin America and the Caribbean (ECLAC), the region's economy will grow by $5.2 \%$ in 2021 . ECLAC mentions that by 2022 it will only reach $2.9 \%$, thereby increasing extreme poverty -a determining factor in the increase in hunger-. In this article, it is presented how backyard agriculture allows to supply food for self-consumption in Mexican families, allowing the surplus -yes, it exists-, to be commercialized; allowing to generate an economic income to the family. The scope of the study is exploratory on the habits of the families of the Bajío; the study carried out shows that it is women who promote the cultivation of vegetables and fruit trees, to obtain healthy and fresh food; and in this sense, pay to guarantee food security. From the above, it follows that precision backyard agriculture promoted by Mexican women is a viable tactical strategy, which contributes to the socioeconomic reactivation in post-COVID-19 times.
\end{abstract}

Keywords: Backyard agriculture, peasant women, socioeconomic reactivation, post COVID-19, food security.

\title{
Introducción
}

La emergencia sanitaria originada por la pandemia del COVID-19, generó un desequilibrio en diversas cadenas de valor, dada la interrupción y la reducción de su sinergia; e.g., algunas empresas manufactureras del acero pararon su producción afectando a la industria de la construcción, la automotriz, entre otras. Esta irrupción ocasionó que las cadenas de valor se desincronizaran en algunos, dichas cadenas se fracturaron-.

El Banco Mundial, establece que el impacto de la pandemia a nivel mundial, en el empleo no ha tenido precedente; son los gobiernos, quienes están desarrollando y adoptando medidas para mitigar el impacto negativo de la crisis de la salud en las empresas y las personas, y así reactivar lo más pronto la economía (World Bank, 2021). La emergencia sanitaria derivada del COVID19 , ha ocasionado una reducción en el bienestar alrededor del mundo -mayor mortalidad y mayor pobreza-; se estima que a causa del COVID-19 se perdió casi 20 millones de años de vida esto para el 2020, y que, durante el mismo período se pasaron más de 120 millones de años adicionales en la pobreza; por lo que, la carga de pobreza de la pandemia, en relación con la carga de mortalidad, es mucho mayor para los países pobres (Ferreira et al., 2021). Las consecuencias económicas de la crisis ya están alcanzando niveles catastróficos (United Nations, 2020).

Según (United Nations Development Programme, 2021), la pandemia de COVID-19 es mucho más que una crisis de salud, se está afectando a 


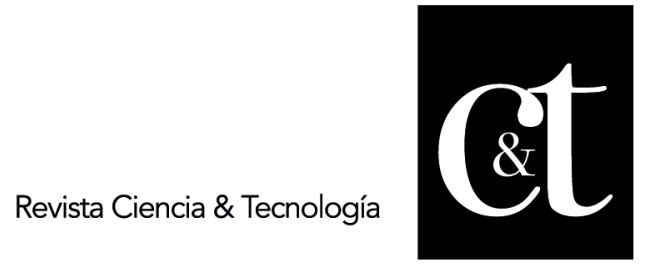

No. 33, 31 de enero de 2022

ISSN impreso: 1390 - 6321

ISSN online: 2661 - 6734

sociedades y economías en su núcleo; ahora bien, si bien es cierto que el impacto de la pandemia varia de un país a otro, es altamente probable que aumente la pobreza y las desigualdades a escala mundial; por lo que, el logro de los Objetivos de Desarrollo Sostenible (ODS), sea aún más urgente. En México, la pandemia pone en mayor vulnerabilidad a las mujeres y niñas; existen datos que indican que son las mujeres las principales responsables de obtener y cocinar alimentos para la familia (Sulmont et al., 2020).

En este orden de ideas, la cadena agroalimentaria también se vio afectada por los efectos de la pandemia. La producción industrial de alimentos se ha visto afectada. Por lo que, la hambruna durante y posterior al COVID-19 podría convertirse en la próxima pandemia que enfrenten las sociedades (Enríquez \& Sáenz, 2021). Es en el sector agroalimentario, en donde, los inéditos problemas han dado lugar a inéditos desafíos; en América Latina y el Caribe (ALyC), no se ha detenido la producción de alimentos - los alimentos son esenciales(CEPAL \& FAO, 2020). Históricamente, en periodos de recuperación económica como la actual-, los hogares han complementado la dieta familiar mediante el huerto traspatio (Burgin, 2018). Ghosh (2021), establece que, a través de los huertos familiares se genera resiliencia, se mejora la salud y la seguridad alimentaria, al mismo tiempo en que se obtienen beneficios ecológicos y ambientales, conectando a las personas con la naturaleza. Entonces, el huerto traspatio expresado como agricultura urbana mejora la resiliencia, la sostenibilidad y la multifuncionalidad (Langemeyer et al., 2021).

Es la agricultura familiar o agricultura traspatio, una estrategia que han adoptado las familias durante la emergencia sanitaria para así cultivar y cosechar su propio alimento, y en todo caso comercializar el excedente -si existe-. Entonces, la agricultura urbana de baja tecnología puede contribuir en gran medida a la autosuficiencia (Hume et al., 2021). La importancia de la agricultura familiar en ALyC está fuera de toda duda, esta representa cerca del $81 \%$ de las explotaciones agrícolas y genera un $50 \%$ del empleo del sector agropecuario en la región (CEPAL \& FAO, 2020).

La agricultura traspatio, ha sido implementada de forma eficiente. En (Nadal et al., 2018), se desarrollan actividades de agricultura urbana para mejorar las dietas, aumentar las áreas verdes, apoyar las economías locales y reducir las emisiones de $\mathrm{CO}_{2}$; en Oakland, California los terrenos baldíos son empleados para la producción y el consumo de hortalizas urbanas (McClintock et al., 2013); en (Amiri et al., 2021), se evalúa como los huertos escolares son una herramienta educativa eficaz en lugar de una mera herramienta para promover la actitud, el conocimiento y la experiencia ambiental; Vannozzi Brito y Borelli (2020), muestran el potencial de los bosques urbanos para mejorar la seguridad alimentaria; en (Djokić et al., 2018; He \& Zhu, 2018), los huertos comunitarios informales han brindado a los productores la oportunidad de ahorrar gastos y proveer de alimentos saludables, mejorando la calidad de vida; en Australia, la agricultura urbana tiene el potencial de mitigar los efectos de los extremos del cambio climático, al mismo tiempo, en que se proporciona múltiples beneficios como mejorar el bienestar, las conexiones entre la gente y la naturaleza, y la seguridad alimentaria (Langemeyer et al., 2021); en (Santos et al., 2018), el desarrollo rural en el estado brasileño de Minas Gerais se alcanza mediante la agricultura familiar; según Mărunţelu (2020), esta práctica 


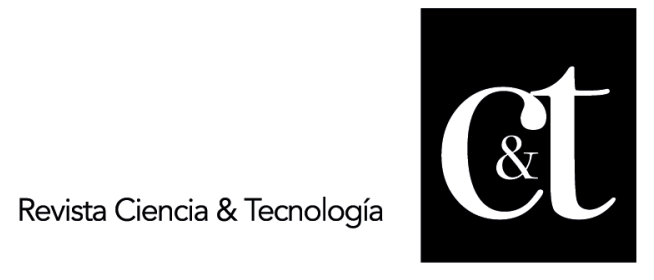

No. 33, 31 de enero de 2022

ISSN impreso: 1390 - 6321

ISSN online: 2661 - 6734

permitió alcanzar una agricultura sostenible en Rumania; además, los huertos familiares pueden mejorar la seguridad alimentaria, la diversidad, el valor nutritivo y el microambiente alrededor del hogar familiar (Lal, 2020).

La producción de esta práctica agrícola se ha optimizado. En (Tong et al., 2020), un nuevo modelo de optimización espacial se emplea para optimizar la producción alimentaria urbana en Tucson, Arizona; en (Galvão et al., 2018), presenta un mecanismo de dosificación de fertilizantes; mientras que Marcelino et al. (2018), se diseñó un prototipo con Raspberry Pi (RPi) y Arduino en conjunto con sensores y actuadores, y así apoyar en la agricultura de precisión de baja escala.

Aunado a esto, es posible ubicarlo como parte de un sistema de autoconsumo; e.g., en (Suárez-Cáceres et al., 2021), se evalúa la producción acuapónica de un policultivo de diferentes hortalizas, hierbas y frutas junto con tilapia, con fines de autoconsumo.

A partir de lo anteriormente expuesto, el presente estudio de investigación persigue evaluar la potencialidad de la producción agrícola representada mediante el huerto traspatio como estrategia táctica que permita la reactivación socioeconómica de la sociedad mexicana durante y después de la pandemia, a partir del desarrollo de esta práctica en manos de las mujeres jefas de familia-, permitiendo proveer a la familia de un alimento sano y fresco como complemento a la dieta alimenticia, al mismo tiempo - si es que existe, el excedente sea comercializado y así obtener un beneficio económico.

El estudio considera que el liderazgo de la mujer mexicana en la actividad agrícola expresada como agricultura traspatio, tiene la capacidad de proveer alimento para autoconsumo y generación de ingreso por la venta del excedente, con lo que, se abona a garantizar la seguridad alimentaria de la familia mexicana, al mismo tiempo en que es posible la reactivación económica de la sociedad mexicana en la presente pandemia a la familia mexicana.

\section{Metodología}

El presente estudio aborda la aplicación de un cuestionario a 384 familias mexicanas de la llamada Región del Bajío -región de tierras bajas, considerando los estados de Guanajuato, Querétaro, Aguascalientes, zona oriente de Jalisco, zona norte de Michoacán, y San Luis Potosí-, habiendo sido considerada como el granero del país por el alto rendimiento de sus cosechas de granos básicos debido a la calidad de sus tierras. La Región del Bajío es considerada como objeto de estudio, ya que esta zona presenta un crecimiento del $5.3 \%$ anual. En tal sentido, el estudio es considerado exploratorio ya que persigue identificar las prácticas agrícolas que son y han sido empleadas por las familias de la región bajo estudio, para satisfacer las necesidades complementarias de alimentación para autoconsumo.

La Figura 1, presenta la metodología empleada para el desarrollo de la investigación. Se destaca que la determinación de las 384 familias a ser 
encuestadas, se desprende al emplear un nivel de confianza $(\alpha)$ del $95 \%$, con una probabilidad de éxito $(p)$ del $50 \%$ y una probabilidad de fracaso $(q)$ de su complemento, el 50\%.

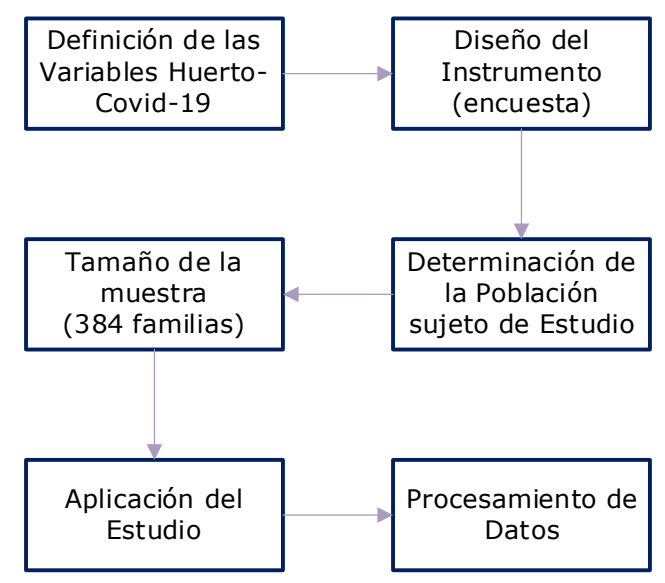

Figura 1: Metodología para la determinación de los hábitos de cultivo Fuente: Elaboración propia

\section{Resultados y discusión}

Las variables de interés que se considera influyen en el huerto traspatio como consecuencia de la pandemia del COVID-19, e.g., necesidad de cultivo para autoconsumo, comportamiento de los integrantes de la familia en la participación del cultivo, cuidado y cosecha del producto agrícola, determinación de los tipos de cultivo: frutas, verduras, hortalizas; se plasmaron en un instrumento, cuestionario (véase Figura 2).

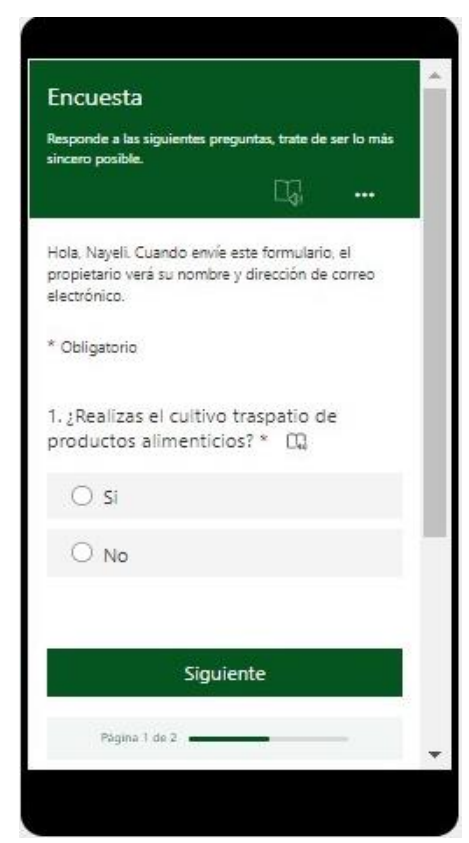

Figura 2: Vista del cuestionario en Smartphone Fuente: Elaboración propia 
La aplicación del cuestionario evidenció que los efectos de la pandemia, han impactado negativamente en la economía de las familias mexicanas (ver Figura $3)$.

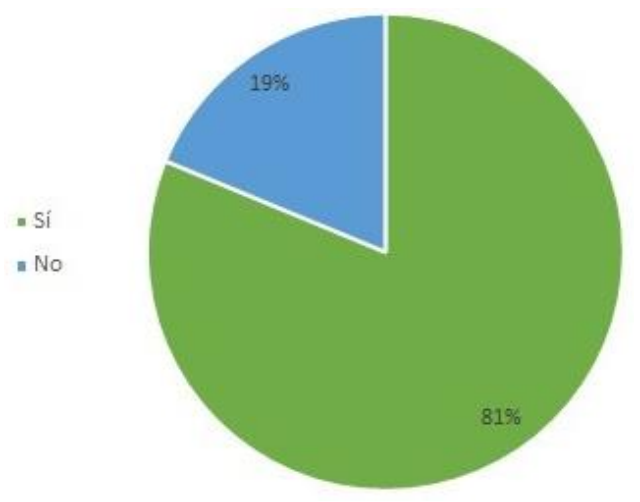

Figura 3: Impacto de las afectaciones de la pandemia en la alimentación Fuente: Elaboración propia

La Figura 4, presenta que el $76 \%$ de la población encuestada indico que inicio el cultivo en huerto traspatio dado el contexto de la emergencia sanitaria, donde los protocolos de salud estipulaban restricción de movilidad, lo que complicó el acceso en la adquisición de productos agrícolas, sanos y frescos; aunado, a este contexto el desempleo, derivado del cierre parcial o total impacto directamente al ingreso económico de la familia.

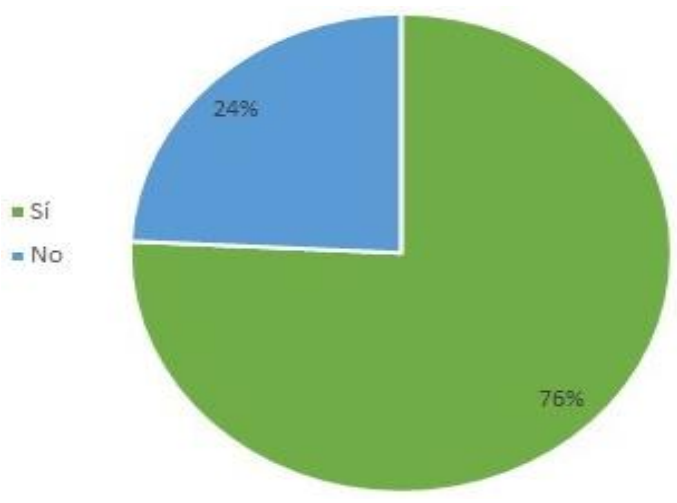

Figura 4: Práctica de huerto traspatio a partir de la pandemia Fuente: Elaboración propia

Dado este contexto, se tuvo la necesidad de autoproducción (ver Figura 5). Siendo las mujeres las impulsadoras de esta práctica, tal y como se muestra en la Figura 6. 


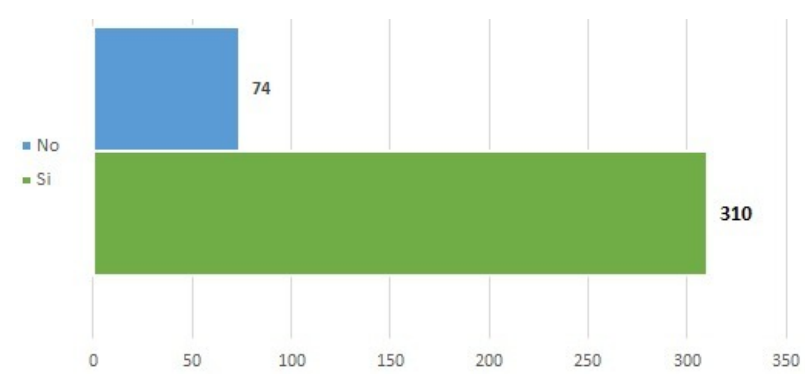

Figura 5: Necesidad de autoproducción Fuente: Elaboración propia

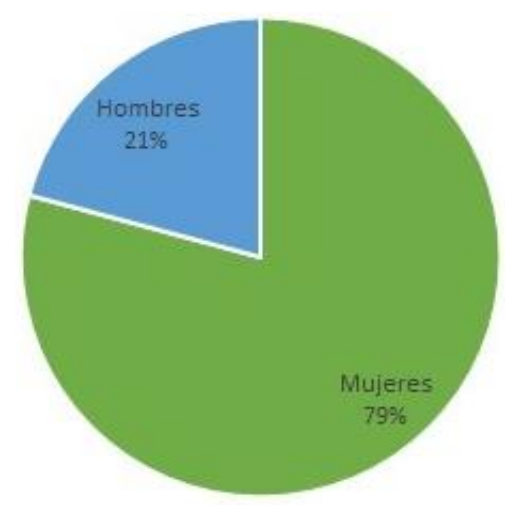

Figura 6: Integrante de la familia como líder/responsable del huerto traspatio

Fuente: Elaboración propia

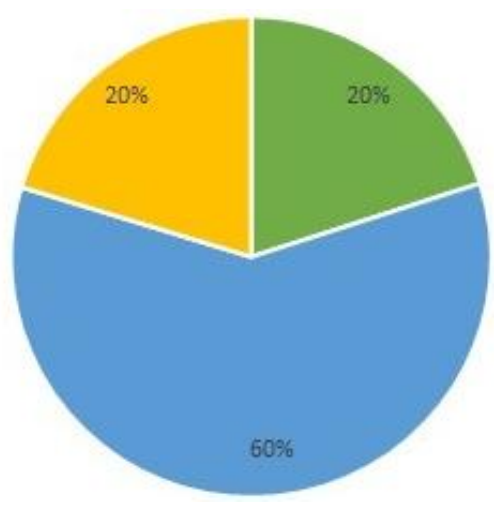

Figura 7: Rango de edad de los responsables del huerto traspatio Fuente: Elaboración propia 


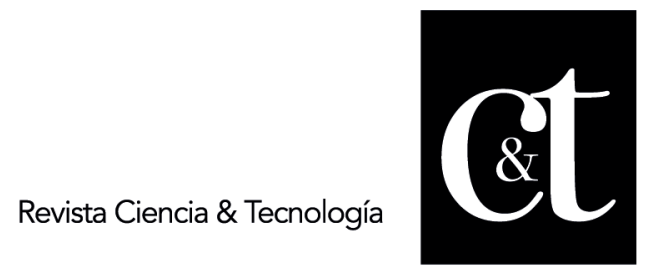

No. 33, 31 de enero de 2022

ISSN impreso: 1390 - 6321

ISSN online: 2661 - 6734
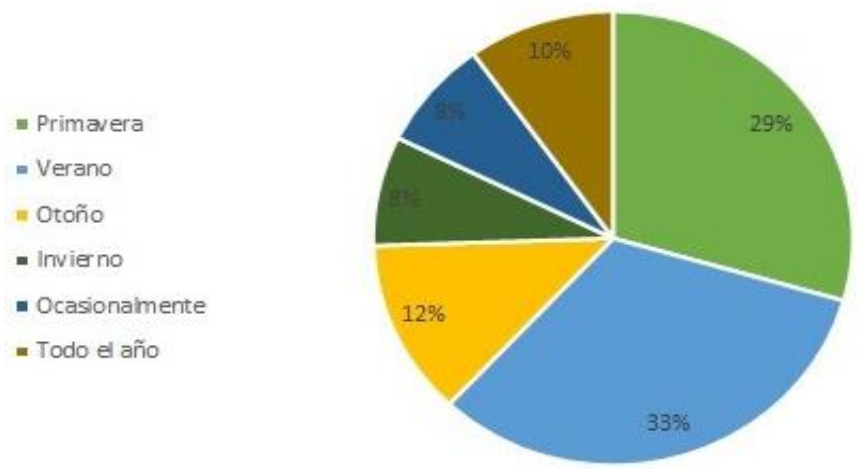

Figura 8: Temporada de cosecha

Fuente: Elaboración propia

Siendo el principal objetivo el autoconsumo y la venta (ver Figura 9).

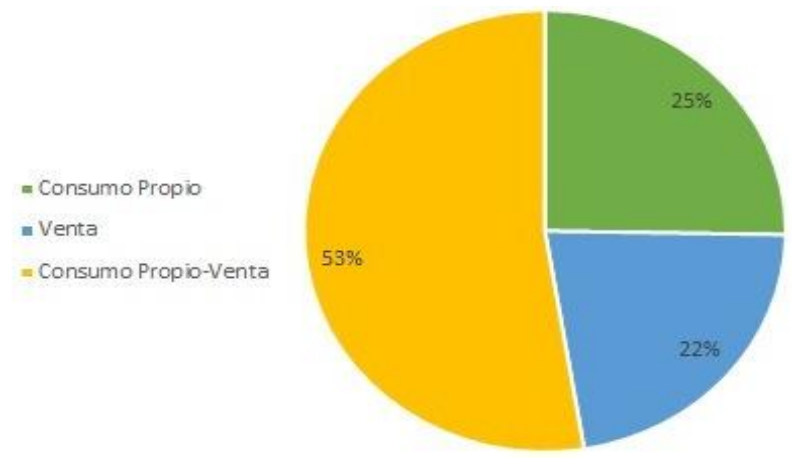

Figura 9: Comportamiento del destino final del cultivo Fuente: Elaboración propia

Ahora bien, las principales hortalizas-verduras son el camote, seguida de la zanahoria (ver Figura 10a); mientras que en las frutas de preferencia es posible ubicar a la manzana (ver Figura 10b); finalmente, como otros cultivos predomina el frijol (ver Figura 10c).

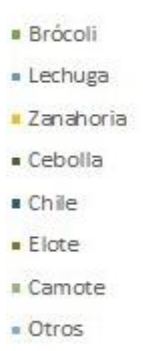

a) Tipos de hortalizas-verduras cosechadas

Montalvo-Romero, Montero Rosales, Merino-Flores, Tavares-López. Potencialidad de la agricultura traspatio de precisión como estrategia táctica en la reactivación: mujeres mexicanas 


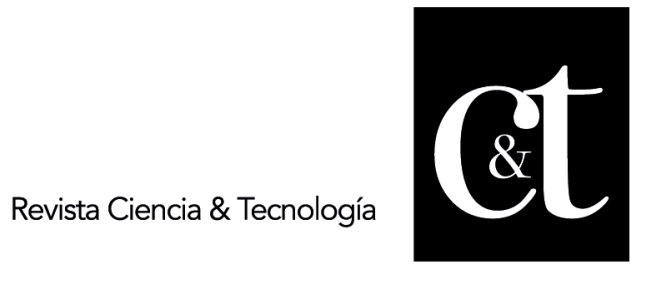

No. 33, 31 de enero de 2022

ISSN impreso: 1390 - 6321

ISSN online: 2661 - 6734

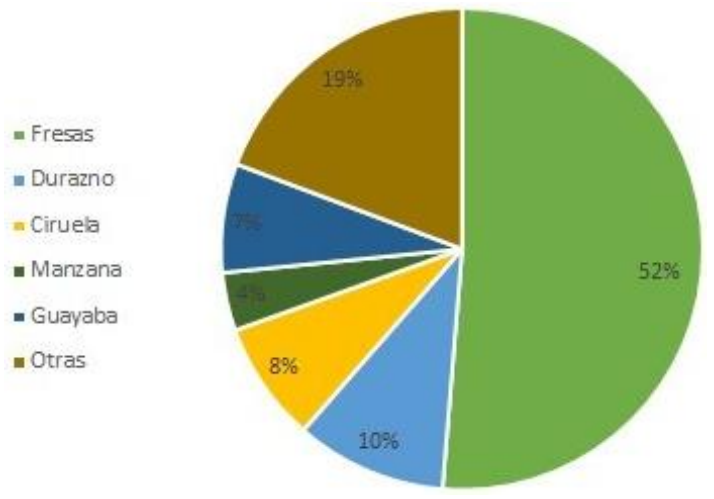

b) Tipos de frutas cosechadas

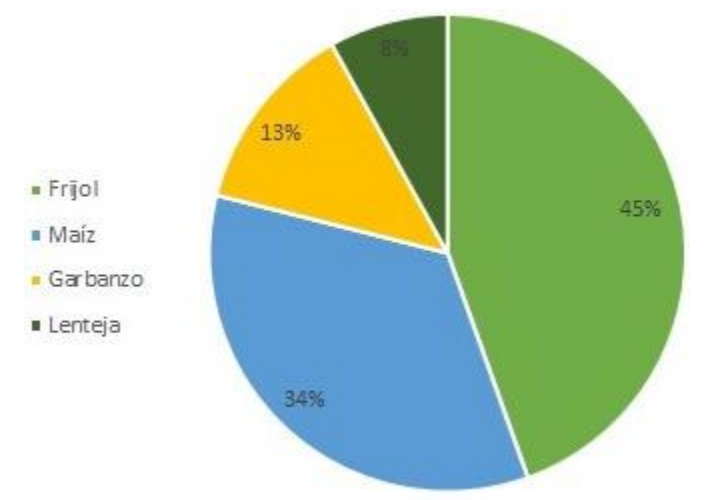

c) Otros cultivos cosechados

Figura 10: Principales cultivos en la Región del bajío

Fuente: Elaboración propia

Entonces, la pandemia por COVID-19 ha representado afectaciones en todos los sectores, siendo uno de los estragos más impactantes la economía de las familias. Sin embargo, el contexto de la emergencia sanitaria esta impulsando el desarrollo de actividades que estaban olvidadas, como lo es el cultivo traspatio. La resiliencia de aquellas familias, especialmente de mujeres, de la necesidad de alimentación impulso a crear estrategias para obtener alimento propio y en el mejor de los casos obtener el ingreso.

La situación de la pandemia debe verse más allá que una afectación negativa, debe plantearse como una alternativa que de ser aprovechada de forma adecuada tiene - dentro de la reactivación hacia la nueva normalidad- la capacidad, de crear sociedades más resilientes, sustentables y sostenibles; por lo que, el desarrollo de estrategias, e.g., políticas gubernamentales, programas, entre otros, es crítico.

Los resultados del estudio muestran que el desarrollo de estrategias que giren en torno a la producción agrícola soportada en la mujer como jefa de familia es viable y factible.

\section{Conclusiones}

Del presente estudio se desprende que el enfoque metodológico empleado en el presente estudio exploratorio, para identificar la estrategia que las familias mexicanas -específicamente las radicadas en la Región del Bajío-, ha permitido identificar y con ello caracterizar el modo en que las familias han hecho fuerte al fenómeno atípico del desabasto de alimentos, debido a la fractura y desincronización de la cadena agroalimentaria, como consecuencia de la pandemia por COVID-19. En tal sentido, el estudio evidencia que la capacidad de subsistencia, de las familias mexicanas es lograda por medio de la agricultura traspatio. Por lo que, esta práctica agrícola táctica permite solventar la necesidad de autoalimento, y con ello abonar a garantizar la 


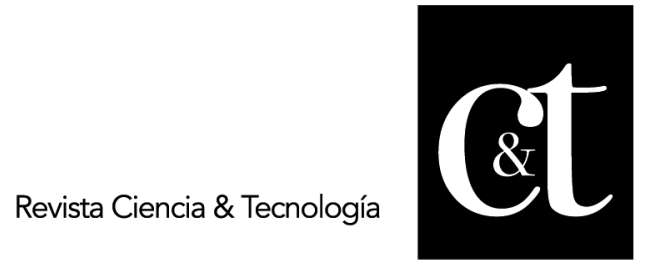

No. 33, 31 de enero de 2022

ISSN impreso: 1390 - 6321

ISSN online: 2661 - 6734

seguridad alimentaria de las familias implicadas, mientras que los excedentes permiten generar un ingreso a partir de la comercialización de dicho producto.

Entonces, el estudio permitió identificar la potencialidad de los huertos traspatio como estrategia táctica en la reactivación que ha sido elegido por las mujeres mexicanas de la Región del Bajío, la cual, mediante la Agricultura de Precisión puede ser optimizada, y por consiguiente un mejor producto agrícola será obtenido. El enfoque metodológico empleado en el presente estudio, evidencia que la encuesta es un instrumento potencial para la caracterización de fenómenos de la sociedad. Como trabajo futuro, se tiene que, con los datos obtenidos, se creará un estudio que permita estandarizar los procesos de cultivos, y con ello apoyar a generar el mayor rendimiento de los cultivos, así mismo determinar los nutrimientos en cantidades necesarias que permitan obtener el mayor rendimiento de los cultivos. Finalmente, los hallazgos del presente estudio sientan las bases para el desarrollo de proyectos que permitan el impulso de sistemas agroalimentarios para autoconsumo basados en huertos traspatio a partir de la gestión de las mujeres como motor transformacional.

\section{Referencias}

Amiri, A., Geravandi, S., \& Rostami, F. (2021). Potential effects of school garden on students' knowledge, attitude and experience: A pilot project on sixth grade students in Iran. Urban Forestry \& Urban Greening, 62, 127174. https://doi.org/10.1016/J.UFUG.2021.127174

Burgin, S. (2018). 'Back to the future'? Urban backyards and food selfsufficiency. Land Use Policy, 78, 29-35. https://doi.org/10.1016/J.LANDUSEPOL.2018.06.012

CEPAL, \& FAO. (2020). Sistemas alimentarios y COVID-19 en América Latina y el Caribe: Impactos y oportunidades en la producción de alimentos frescos.

Djokić, V., Ristić Trajković, J., Furundžić, D., Krstić, V., \& Stojiljković, D. (2018). Urban garden as lived space: Informal gardening practices and dwelling culture in socialist and post-socialist Belgrade. Urban Forestry \& Urban Greening, 30, 247-259. https://doi.org/10.1016/J.UFUG.2017.05.014

Enríquez, A., \& Sáenz, C. (2021). Primeras lecciones y desafíos de la pandemia de COVID-19 para los países del SICA. www.cepal.org/apps

Ferreira, F. H. G., Sterck, O., Mahler, D., \& Decerf, B. (2021). Death and Destitution The Global Distribution of Welfare Losses from the COVID-19 Pandemic. http://www.worldbank.org/prwp. 


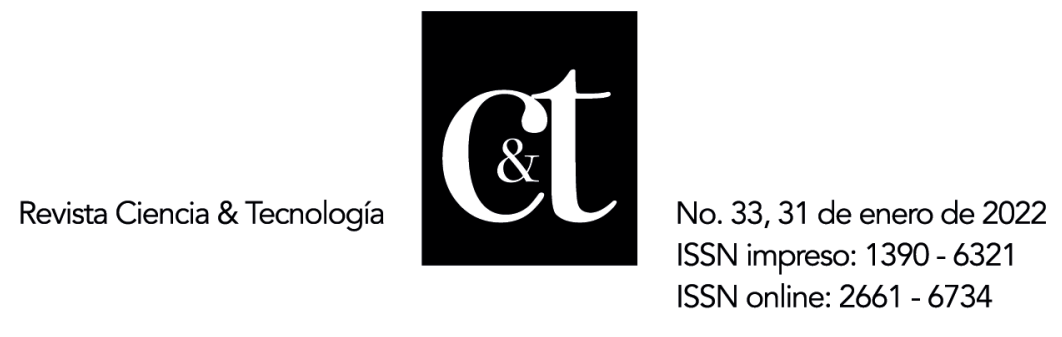

Galvão, C. B., Albiero, D., Garcia, A. P., \& Monteiro, L. de A. (2018). Fertilizer metering mechanism with helical conic cylindrical thread for family agriculture. Engenharia Agrícola, 38, 934-940.

Ghosh, S. (2021). Urban agriculture potential of home gardens in residential land uses: A case study of regional City of Dubbo, Australia. Land Use Policy, 109, 105686. https://doi.org/10.1016/J.LANDUSEPOL.2021.105686

He, B., \& Zhu, J. (2018). Constructing community gardens? Residents' attitude and behaviour towards edible landscapes in emerging urban communities of China. Urban Forestry \& Urban Greening, 34, 154-165. https://doi.org/10.1016/J.UFUG.2018.06.015

Hume, I. v., Summers, D. M., \& Cavagnaro, T. R. (2021). Self-sufficiency through urban agriculture: Nice idea or plausible reality? Sustainable Cities and Society, 68, 102770. https://doi.org/10.1016/J.SCS.2021.102770

Lal, R. (2020). Home gardening and urban agriculture for advancing food and nutritional security in response to the COVID-19 pandemic. Food Security, $1-6$.

Langemeyer, J., Madrid-Lopez, C., Mendoza Beltran, A., \& Villalba Mendez, G. (2021). Urban agriculture - A necessary pathway towards urban resilience and global sustainability? Landscape and Urban Planning, 210, 104055. https://doi.org/10.1016/J.LANDURBPLAN.2021.104055

Marcelino, R., Casagrande, L. C., Cunha, R., Crotti, Y., \& Gruber, V. (2018). Internet of Things applied to precision agriculture. In Online Engineering \& Internet of Things (pp. 499-509). Springer.

Mărunțelu, I. (2020). Research on the small peasant individual households in Romania within the framework of sustainable agriculture. Scientific Papers Series-Management, Economic Engineering in Agriculture and Rural Development, 20(1), 341-346.

McClintock, N., Cooper, J., \& Khandeshi, S. (2013). Assessing the potential contribution of vacant land to urban vegetable production and consumption in Oakland, California. Landscape and Urban Planning, 111(1), 46-58. https://doi.org/10.1016/J.LANDURBPLAN.2012.12.009

Nadal, A., Cerón-Palma, I., García-Gómez, C., Pérez-Sánchez, M., RodríguezLabajos, B., Cuerva, E., Josa, A., \& Rieradevall, J. (2018). Social perception of urban agriculture in Latin-America. A case study in Mexican social housing. Land Use Policy, 76, 719-734. https://doi.org/10.1016/J.LANDUSEPOL.2018.02.055

Santos, L. F. dos, Ferreira, M. A. M., \& Campos, A. P. T. de. (2018). Rural development and family agriculture in the Brazilian state of Minas Gerais in the light of multivariate data analysis. Interações (Campo Grande), 19, 827843. 


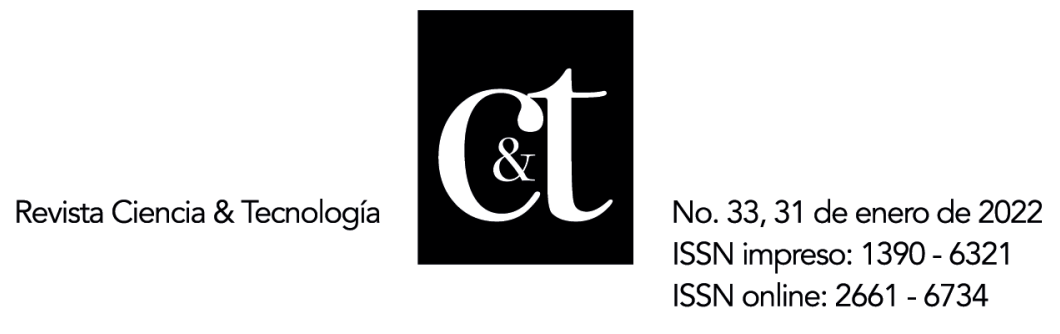

Suárez-Cáceres, G. P., Lobillo-Eguíbar, J., Fernández-Cabanás, V. M., Quevedo-Ruiz, F. J., \& Pérez-Urrestarazu, L. (2021). Polyculture production of vegetables and red hybrid tilapia for self-consumption by means of microscale aquaponic systems. Aquacultural Engineering, 95, 102181. https://doi.org/10.1016/J.AQUAENG.2021.102181

Sulmont, A., Martínez, C., García De Alva, M., Correa, A., Leal, V., Mendoza, O., Visser, S., \& Pinelo, A. (2020). Desafíos de desarrollo ante la COVID-19 en México. Panorama Socioeconómico. www.latinamerica.undp.org

Tong, D., Crosson, C., Zhong, Q., \& Zhang, Y. (2020). Optimize urban food production to address food deserts in regions with restricted water access. Landscape and Urban Planning, 202, 103859. https://doi.org/10.1016/J.LANDURBPLAN.2020.103859

United Nations. (2020). Responding to COVID-19 and Recovering Better: $A$ Compilation by UN DESA.

United Nations Development Programme. (2021). Socio-economic impact of COVID-19. https://www.undp.org/coronavirus/socio-economic-impactcovid-19

Vannozzi Brito, V., \& Borelli, S. (2020). Urban food forestry and its role to increase food security: A Brazilian overview and its potentialities. Urban

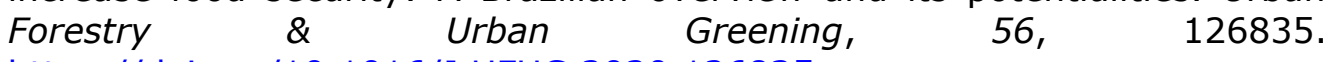
https://doi.org/10.1016/J.UFUG.2020.126835

World Bank. (2021). COVID-19: Development news, research, data. COVID19 (Coronavirus) Response. https://www.worldbank.org/en/topic/financialsector/coronavirus

En relación a las percepciones de las estrategias inbound marketing, son medidas a través de un cuestionario multifactor, que es aplicable a través de una encuesta diseñada para medir las variables según las dimensiones consideradas. 
No. 33, 31 de enero de 2022

ISSN impreso: 1390 - 6321

ISSN online: 2661 - 6734 